\title{
The higher gastrin levels were associated with better glycemic
}

\section{control}

Georgios Boutzios' ${ }^{1}$ Krystallenia Alexandraki¹, Panayiotis Moschouris ${ }^{1}$, Eleni Lampropoulou ${ }^{1}$, Anastasia Evaggelatou² Antigoni Velidaki², Eufrosyni Kitsou², Gregory Kaltsas ${ }^{1}$

${ }^{1}$ Endocrine Oncology Unit, Section of Endocrinology, Department of Pathophysiology, National and Kapodestrian University of Athens Medical School, ATHENS, Greece 2Department of Nuclear Medicine, Laiko University Hospital, ATHENS, Greece

\section{Introduction}

Gastrin is an early incretin candidate, since it is released by oral glucose and potentiates the glucose- induced insulin secretion. It has been shown that only in hypoglycemic or hyperglycemic conditions gastrin release is influenced by changes in blood glucose and insulin concentrations

\section{$\operatorname{Aim}(\mathbf{s})$}

To evaluate whether there is a relation between fasting serum glucose levels, HbA1c, and serum gastrin concentrations

\section{Materials and methods}

Gastrin and glucose levels and HbA1c were measured in 386 blood samples. We studied gastrin serum concentrations dividing the samples in 3 subgroups with glucose concentrations $<100 \mathrm{mg} / \mathrm{dl}(A), 101-200 \mathrm{mg} / \mathrm{dl}(B),>201 \mathrm{mg} / \mathrm{d}(C)$, in 3 subgroups with $\mathrm{HbA1c}<5.7 \%$ (a), $5.7-6.4 \%$ (b), >6.5\% (c) and in 3 subgroups with gastrin levels 100-200 ng/ml (I), 201$500 \mathrm{ng} / \mathrm{ml}$ (II) and $>500 \mathrm{ng} / \mathrm{ml}$ (III).

\section{Results}

Gastrin and $\mathrm{HbA1C}$ were negatively correlated $(r=-0.32$, $p=0.001$ ) in the total population of samples studied.

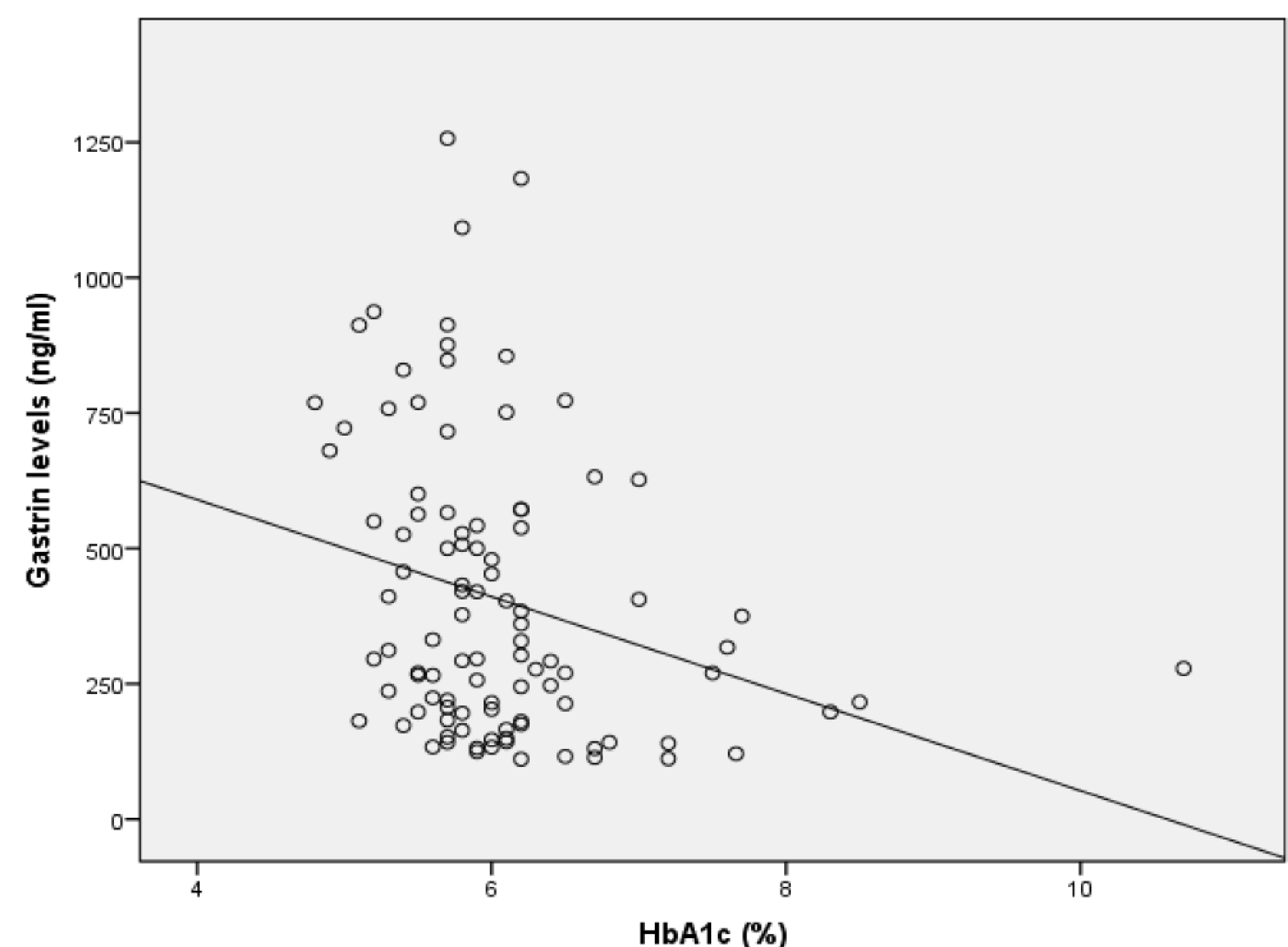

In the subgroups of normal, prediabetic, diabetic range of $\mathrm{HbA1c}$, gastrin levels differed between normal (a) and prediabetic (b) range $(p=0.05)$ and prediabetic $(b)$ and diabetic (c) range of $\mathrm{HbA1c}(p=0.005)$.

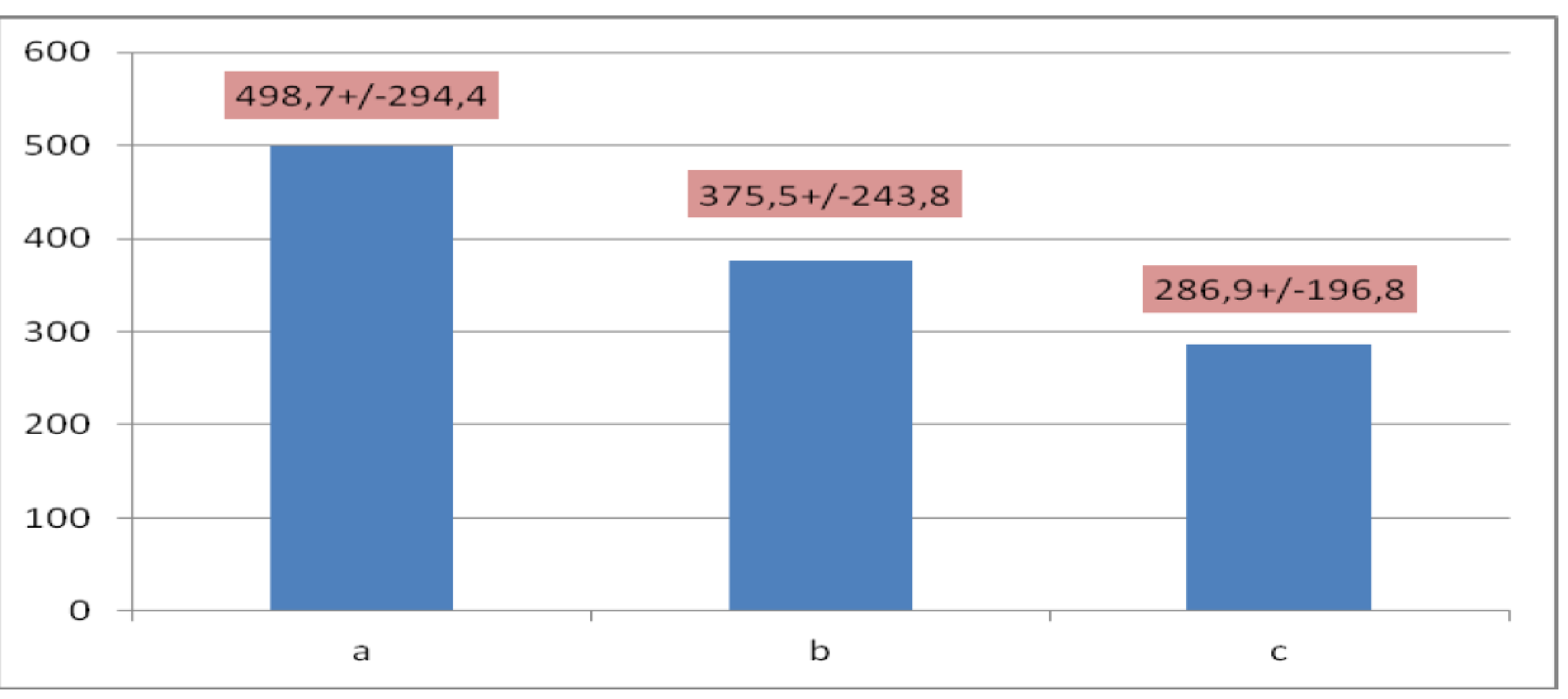

Gastrin levels in 3 subgroups of HbA1c levels

\section{Results}

No difference was found in gastrin concentration in the subgroups of normal, moderately increased, increased glucose concentrations.

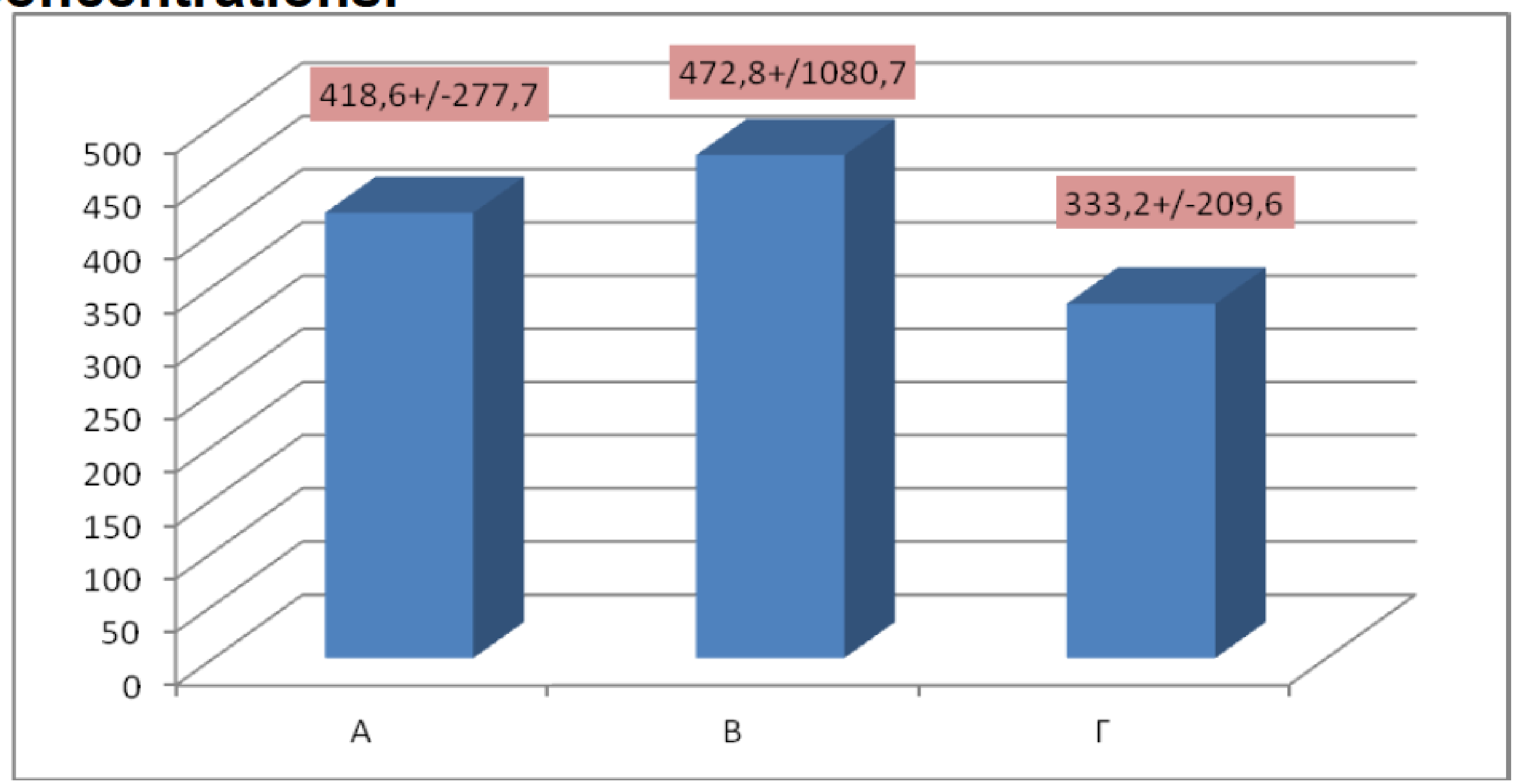

Gastrin levels in 3 subgroups of glucose levels

In low, mid-high, high gastrin levels glucose levels differed only between moderate (II) and high (III) gastrin levels $(p=0.05)$.

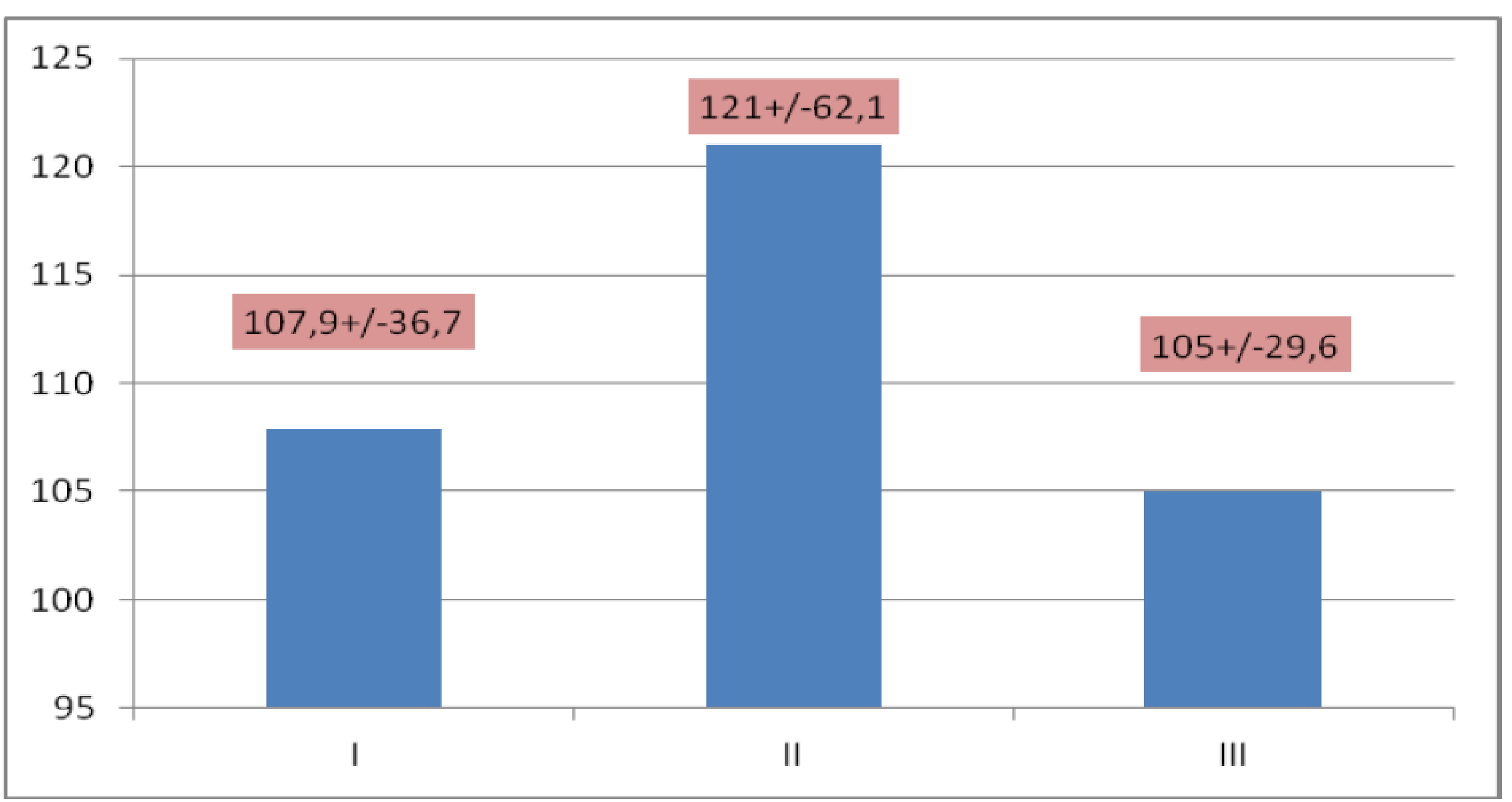

Glucose levels in 3 subgroups of Gastrin levels

In low, mid-high, high gastrin levels HbA1c values differed between low (I) and high (III) $(p=0.006)$ and moderate (II) and high (III) $(p=0.001)$ gastrin levels.

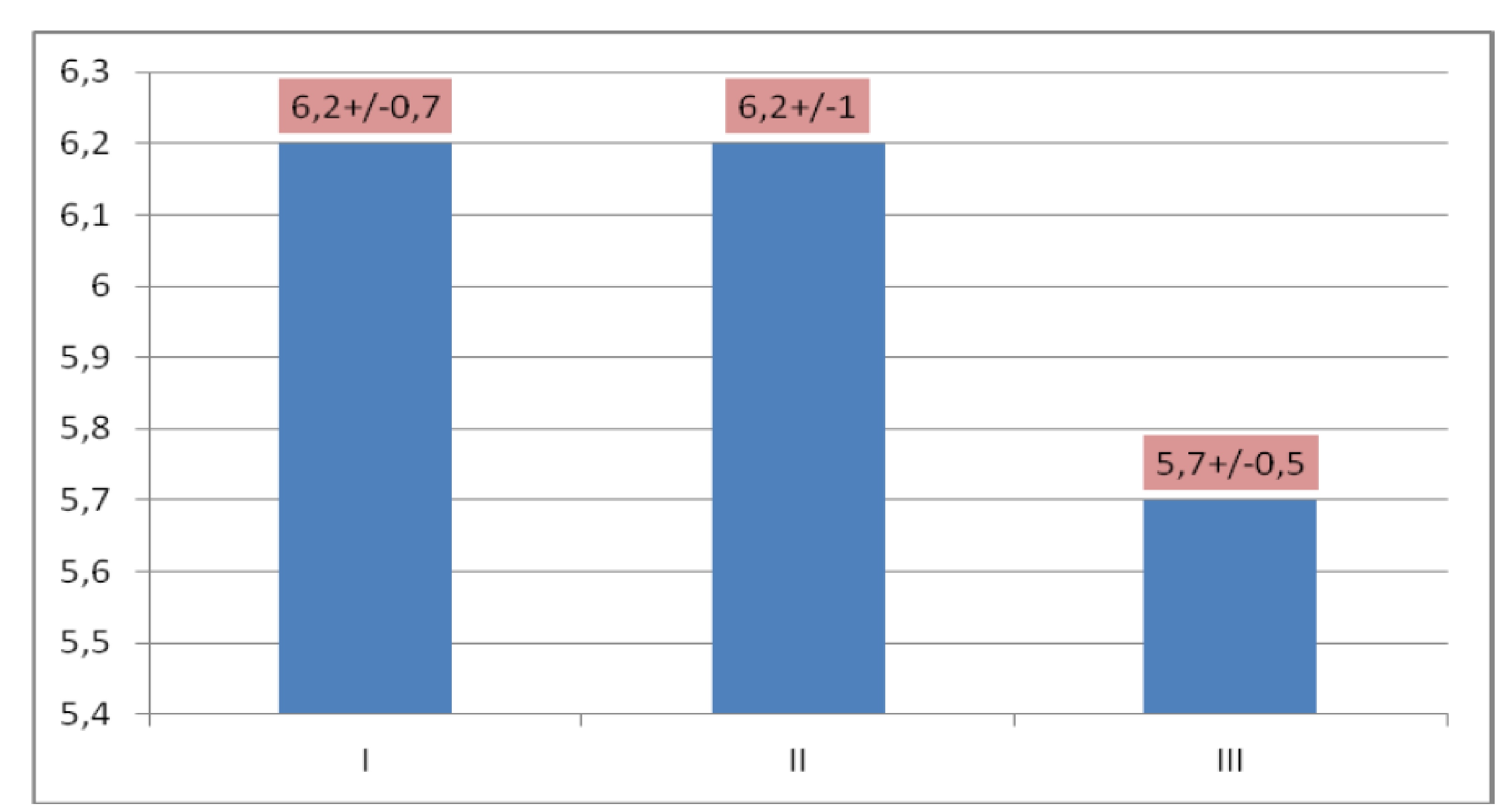

HbA1c levels in 3 subgroups of Gastrin levels

\section{Conclusions}

A negative correlation between $\mathrm{HbA1c}$ and gastrin levels has been shown in the present study. In patients with higher gastrin levels had lower HbA1c compared to patients with high or moderate gastrin levels, indicating a better glycemic control. Nevertheless further studies are needed to confirm these findings.

\section{References}

Hove KD, et al. Diabetologia. 2013;56:22-30

Hove KD, et al. Diabetes Res Clin Pract. 2010;90:e72-4.

Barchetta I, et al. Acta Diabetol. 2015 Feb 27.

Han N, et al. Can J Diabetes. 2015;39:24-8 